\title{
Distribución de fotoasimilados en los órganos vertederos de plantas Solanaceas, caso tomate y papa. Una revisión
}

\author{
Distribution of photoassimilates in sink organs of plants of Solanaceas, \\ tomato and potato. A review
}

\author{
Andrés F. León-Burgos ${ }^{1}$ \\ Gina Yuret Beltrán Cortes ${ }^{2}$ \\ Andrea Liliana Barragán Pérez ${ }^{3}$ \\ Helber Enrique Balaguera-López ${ }^{4}$
}

DOI: https://doi.org/10.19053/01228420.v18.n3.2021.13566

RESUMEN: Los cultivos de tomate y papa son productos agrícolas de gran importancia a nivel mundial por su valor nutricional e industrial. La distribución de fotoasimilados en los órganos vertederos de estos cultivos depende en gran medida del metabolismo y partición de carbono generado en los procesos fotosintéticos, en la dinámica tanto de los órganos fuente y vertedero como en la actividad de enzimas vinculadas al metabolismo de azúcares, así como factores ambientales y nutricionales. Basado en esto, el objetivo de este documento fue revisar y discutir sobre el conocimiento actual de la distribución de los fotoasimilados en los órganos vertederos y los factores que pueden afectar este mecanismo en los cultivos de tomate y papa. De acuerdo con la información recopilada en artículos recientes de investigación y revisión, se reporta que alrededor del $80 \%$ o $90 \%$ de los fotoasimilados producidos en los órganos fuentes en los cultivos de tomate y papa son transportados o translocados hacia los órganos vertederos de interés comercial (frutos y tubérculos). Tanto el desbalance en fuente/vertedero, así como el estrés hídrico y nutricional, en especial, deficiencias de nitrógeno y potasio afectan significativamente la distribución y transporte de los fotoasimilados. En contraste con los efectos de la calidad de la luz puede mejorar la carga de fotoasimilados en los órganos vertederos y mejorar atributos de la calidad como aumento en el tamaño de los frutos y concentración de azucares. Sin embargo, aún faltan más investigaciones que corrobore este efecto bajo condiciones de campo o en invernadero en las condiciones del trópico.

PALABRAS CLAVE: Fuerza de Vertedero; Relación Fuente/Vertedero; Metabolismo De Azúcares; Raleo De Frutos.
ABSTRACT: Tomato and potato crops are agricultural products of great importance worldwide due to their nutritional and industrial value. The distribution of photoassimilates in the sink organs of these crops depends to a great extent on the metabolism and partition of carbon generated in the photosynthetic processes, on the dynamics of both of the source and sink organs as the activity of enzymes linked to the metabolism of sugars and environmental and nutritional conditions. The objective of this paper was to review and discuss current knowledge of the distribution of photoassimilates in the sink organs and factors that can affect this mechanism in the tomato and potato crops. Based on information collected in recent research and review articles, it is reported that around $80 \%$ or $90 \%$ of the photoassimilates produced in the source organs in tomato and potato crops are transported or translocated to the sink organs of commercial interest (fruits and tubers). Both source/sink imbalance, as well as water stress and nutritional deficiencies, especially nitrogen and potassium significantly affect the distribution of photoassimilates. In contrast to the effects of the quality of light, it can improve the loading of photoassimilates in the sink organs and improve quality attributes of these such as increases in the size and concentration of sugars. However, more research is still needed to corroborate this effect, especially under field or greenhouse conditions in tropical regions.

KEYWORDS: sink strength; source/sink ratio; sugar metabolism; fruit thinning.

FECHA DE RADICACIÓN: 06 de septiembre de 2021 FECHA DE APROBACIÓN: 18 de octubre de 2021

CITAR COMO: León-Burgos, A. F.., Beltrán Cortes, G. Y.., Barragán Pérez, A. L. ., \& Balaguera-López, . H. E. (2021). Distribución de fotoasimilados en los órganos vertederos de plantas Solanaceas, caso tomate y papa. Una revisión. Cien. Agri., 18(3): 79-97. https://doi.org/10.19053/01228420.v1 8.n3.2021.13566

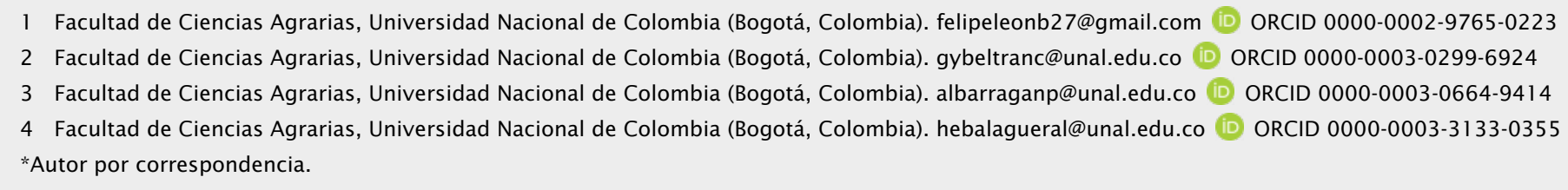




\section{Introducción}

El tomate (Solanum lycopersicum L.) y la papa (Solanum tuberosum L.) son especies de interés económico y hortícola de gran importancia para Colombia, por sus elevados consumos en la canasta familiar y los incrementos en el área cultivable en las últimas décadas (Balaguera-López et al., 2009; Perilla et al., 2011; Córdoba-Novoa et al., 2018; Fedepapa, 2020). Estos cultivos son sembrados en la mayoría de los departamentos de Colombia y están establecidos en sistemas de producción a libre exposición solar, así como en invernaderos o bajo cubiertas plásticas, específicamente, el cultivo de tomate (Ardila et al., 2011; Perilla et al., 2011). Se ha estimado para el año 2019, un área de siembra entre 162,000 a $790,000 \mathrm{ha}^{-1}$ con rendimientos promedio a nivel nacional de $23,000 \mathrm{~kg}$ ha-1 para papa y entre 29,000 a $83,000 \mathrm{~kg}$ ha-1 de tomate tanto a libre exposición solar como bajo invernadero, respectivamente (Agronet, 2019). No obstante, para garantizar la sostenibilidad económica de estos cultivos se ha reportado la necesidad de implementar prácticas adecuadas de manejo agronómico, que tome como base los conocimientos fisiológicos y de esta manera se pueda mejorar tanto la producción como la calidad de los cultivos de tomate y papa (Quintana-Baquero et al., 2010; Perilla et al., 2011; Li et al., 2016).

El proceso de distribución de fotoasimilados en las plantas requiere la formación y translocación continua de compuestos carbonados desde los órganos fuente; definidos como tejidos o órganos autótrofos fotosintéticos, generalmente hojas maduras o bien desarrolladas (Chang et al., 2017; Rodrigues et al., 2019), los cuales, producen carbohidratos para asignarlos a diferentes procesos esenciales en la planta como el crecimiento y desarrollo o translocar estos compuestos hacia los órganos vertederos como hojas en formación, tallos, raíces, flores y frutos (Castellanos et al., 2010; Bihmidine et al., 2013; Osorio et al., 2014; Aluko et al., 2021). Se ha estimado que alrededor del $80 \%$ de los fotoasimilados de las hojas son transportados hacia los órganos vertederos en forma de azúcares solubles, los cuales, proceden de la asimilación de $\mathrm{CO}_{2}$ en las hojas (fotosíntesis) y de procesos de óxidoreducción (metabolismo de azúcar), para que finalmente se movilicen a través del floema hacia los órganos vertederos (Wang et al., 2020; Aluko et al., 2021; López-Delacalle et al., 2021). Sin embargo, este transporte puede ser afectado por condiciones de estrés abiótico y biótico o por el desbalance en la relación de órganos fuente/vertedero.

La manipulación de la relación de órganos fuente/vertedero constituye uno de los principales enfoques de los productores agrícolas que busca garantizar un óptimo desarrollo de las partes cosechables con la calidad deseada (Fischer et al., 2012; Li et al., 2016; Smith et al., 2018; Aslani et al., 2020). Esta situación ha conllevado a centralizar investigaciones en búsqueda de optimizar la producción de fotoasimilados por los órganos fuente (Smith et al., 2018; Rodrigues et al., 2019), y también en mejorar la utilización de los 
fotoasimilados por los órganos vertederos (Bihmidine et al., 2013; Osorio et al., 2014). En varios estudios por medio de la defoliación de hojas o podas de frutos por racimo en cultivos como tomate y papa, se ha constatado los efectos del desbalance de los órganos fuentes/vertedero sobre el desempeño fisiológico de las plantas, y por tanto, en los rendimientos de los cultivos (Castellanos et al., 2010; Golovko et al., 2019; Aslani et al., 2020; Glanz-Idan \& Wolf, 2020).

Los factores ambientales influyen en la relación entre fuente y vertedero (Fischer et al., 2012). Se ha corroborado que factores como características físicas del suelo, la calidad e intensidad de la luz, condiciones de estrés hídrico, temperatura, salinidad y deficiencias nutricionales, pueden tener efectos perjudiciales en el balance de las relaciones entre órganos fuentes/vertederos en los cultivos de tomate y papa (Balaguera-López et al., 2009; Li et al., 2016; Aliche et al., 2020; Ji et al., 2020; López-Delacalle et al., 2021). De hecho, se ha reportado que los factores mencionados anteriormente afectan los procesos fotosintéticos, la partición de carbono, las concentraciones de azúcares, la expresión génica y en general el crecimiento y desarrollo de las plantas de tomate y papa (Bihmidine et al., 2013; Rodríguez et al., 2016; Glanz-Idan \& Wolf, 2020; Luo et al., 2021). Por lo anterior, el objetivo de este artículo fue realizar una revisión de información sobre los factores que pueden afectar la distribución de fotoasimilados en órganos vertederos, de tal manera que se puedan buscar estrategias para mejorar la cantidad y calidad de los rendimientos en los cultivos de tomate (S. lycopersicum) y papa (S. tuberosum).

\section{Tomate (S. Iycopersicum)}

Los patrones de distribución de fotoasimilados depende en gran medida del crecimiento de las plantas (Li et al., 2015; Osorio et al., 2014). En el cultivo de tomate se obtiene una gran variedad de cultivares con características de crecimiento indeterminado y determinado (Mbonihankuye et al., 2013; Maboko et al., 2017). Para el caso de los cultivares de tipo de crecimiento determinado durante la fase vegetativa los fotoasimilados se translocan para la formación y crecimiento de tallos, hojas y raíces, y posteriormente gran parte de esta se transfiere a las flores y frutos (vertederos) cuando inicia la fase reproductiva. En contraste, con los cultivares de tipo indeterminado, cuando se inicia la fase reproductiva, además de distribuir fotoasimilados en las flores y frutos, también se direccionan hacia las zonas vegetativas de crecimiento activo (meristemos apicales y laterales) lo cual, genera una competencia por la distribución de los fotoasimilados (Quintana-Baquero et al., 2010; Wang et al., 2020). Por ello es importante tener en cuenta la fuerza de los órganos fuentes (hojas maduras), definida como la capacidad de asimilar y convertir el $\mathrm{CO}_{2}$ a azucares y posteriormente exportarlo (Rodrigues et al., 2019) y, por otro lado, la fuerza del vertedero, que es la capacidad para importar, utilizar 
y metabolizar los fotoasimilados (Bihmidine et al., 2013; Chang et al., 2017; Ji et al., 2020).

La fuerza del vertedero de interés económico en el cultivo de tomate son los frutos (Osorio et al., 2014; Ji et al., 2020). Estos órganos tienen la capacidad de importar entre 50 al 90\% de la producción de fotoasimilados de los órganos fuentes y por esto se les considera como vertederos prioritarios (Yoshioka, 1985). Sin embargo, se ha reportado que en las plantas de tomate existe una alta actividad de órganos vertederos, y por tanto, una limitación en los órganos fuentes (Li et al., 2015). De esta manera, en el cultivo de tomate se ha recomendado prácticas de manejo como la poda del meristemo apical y/o raleo de frutos para mejorar la relación de órganos fuente/ vertedero y así obtener una buena producción con frutos de mejor calidad (Quintana-Baquero et al., 2010; Aslani et al., 2020).

\section{Transporte de fotoasimilados hacia órganos vertederos}

Los fotoasimilados translocados hacia los órganos vertederos son procedentes del ciclo de Calvin-Benson-Bassham, en el cual, se obtiene como producto el ácido-3- fosfoglicerato (3-PGA) que puede ser almacenado en formas de almidón en los cloroplastos, o transportados al citosol a través de transportadores específicos (TPT), los cuales, tienen la capacidad de transportar tanto el 3-PGA como fosfatos inorgánicos en modo antiporte (Osorio et al., 2014; López-Delacalle et al., 2021). Después de movilizarse al citosol, el 3-PGA dependiendo de la necesidad de la planta es sintetizado en otros azúcares como hexosas y son asignados para el mantenimiento de procesos esenciales en la planta como síntesis de pared celular, respiración, crecimiento y desarrollo o ser translocado hacia los órganos vertederos en forma de sacarosa por medio de la actividad de enzimas vinculadas en el metabolismo de azucares (invertasa-Inv, sacarosa sintasa-SuSy, sacarosa fosfato sintasaSPS, UDP glucosa, entre otras.) encargadas de la translocación hacia los órganos vertederos (Aluko et al., 2021; Luo et al., 2021; López-Delacalle et al., 2021). Para el cultivo de tomate en condiciones adecuadas, se ha estimado que dentro del $60 \%$ hasta el $80 \%$ de los fotoasimilados producidos en las hojas fuentes son distribuidos hacia los frutos (Yoshioka, 1986).

El proceso de translocación de los fotoasimilados generalmente desde los órganos fuentes hacia los órganos vertederos incluye dos rutas claves. La ruta simplastica donde la sacarosa sintetizada sale de las células del mesófilo y se transfiere a otras células (células acompañantes) a través de los plasmodesmos, siguiendo un gradiente de concentración, o la ruta apoplastica que implica el movimiento de sacarosa a través de proteínas de transporte simporte como SUT o SWEET, las cuales han sido reportadas para las plantas de tomate (Osorio et al., 2014; Ho et al., 2019; Abelanda et al., 2020). Este proceso es considerado transporte activo secundario, el cual, requiere la activación de 
bombas tipo ATPasa por medio de la fosforilación, con el fin de promover el flujo protones $\mathrm{H}^{+}$, para luego ser transportados junto con la sacarosa a las células del parénquima del floema (Carpaneto et al., 2005), en contra de su gradiente de concentración y ser posteriormente descargados en los órganos vertederos (Figura 1) (Bihmidine et al., 2013; Ho et al., 2019). Cuando los azúcares llegan a los órganos vertederos, se convierten a hexosas, y después de varios procesos metabólicos y bioquímicos se sintetizan en almidón como producto de reserva (Chen et al., 2019).

Por otro lado, también se ha reportado el rol del $\mathrm{K}^{+}$sobre la tasa de transporte de fotoasimilados en los frutos de tomate (Mengel \& Viro, 1974; Kanai et al., 2007; Luo et al., 2021). Mengel y Viro (1974) reportan que el $\mathrm{K}^{+}$favoreció entre un $80 \%$ hasta $90 \%$ el transporte de azúcares en mayor medida en la forma de sacarosa. Los roles del $\mathrm{K}^{+}$sobre la tasa de transporte de fotoasimilados han sido asociados al aumento de ATP, dado que este compuesto puede influenciar varios procesos de la translocación hacia los órganos vertederos como la carga de los fotoasimilados dentro del floema (Mengel \& Viro, 1974) y recientemente se ha registrado su función en la actividad de enzimas encargadas del metabolismo de azúcares como SuSy, SPS, SP y adenosin difosfato glucosa pirofosforilasa-AGPasa (Luo et al., 2021). Esta actividad de enzimas es importante debido a que están relacionadas con la fuerza del vertedero, de hecho, se ha relacionado sus funciones en la acumulación de azucares y almidón y en el tamaño del fruto durante la etapa de maduración (Osorio et al., 2014; Luo et al., 2021).

\section{Factores que influyen en la distribución de fotoasimilados en los órganos vertederos}

\section{Relación de órganos fuente/vertedero}

La relación adecuada de fuente/vertedero en tomate está relacionada con la producción y calidad de los frutos (Aslani et al., 2020; Ji et al., 2020). En la Figura la, se puede observar como la carga y transporte de fotoasimilados hacia los frutos de tomate es reducida por la baja actividad de estos órganos (fuerza del vertedero). Esto conlleva a reducir la translocación y utilización de sacarosa hacia los frutos, y por tanto, los fotoasimilados producidos pueden ser almacenados en las hojas fuentes (Li et al., 2015). Sin embargo, esta situación puede alterar negativamente la actividad de la fotosíntesis, dado que se reduce la utilización de 3-PGA y la tasa de carboxilación de la Ribulosa 1,5 bifosfato carboxilasa/oxigenasa (RuBisCo) (Aslani et al., 2020; Fabre et al., 2020).

Para constatar lo anterior, Aslani et al. (2020) manipularon la relación de fuente/ vertedero a través del raleo de frutos de tomate por racimo (alta relación fuente/vertedero). En este estudio, se reportó que el raleo de un fruto por 
cada racimo (1 fruto/racimo), modificó la distribución de los fotoasimilados, la cual, fue asignada y translocada para la formación de nuevas estructuras vegetativas por la baja actividad de la fuerza de vertedero de los frutos. Esto favoreció que se incrementara el tamaño de los frutos no raleados con respecto a las plantas control, además modificó los patrones de partición de la materia seca, enfocándose la distribución hacia los órganos vegetativos y raíces (frutos $20 \%$, tallo $28 \%$, hojas $20 \%$ y raíces $32 \%$ ), con respecto a las plantas control (frutos $43 \%$, tallo 16\%, hojas $26 \% \%$ y raíz 15\%). Así mismo, en este estudio se registró reducciones en las tasas fotosintéticas, las cuales, fueron asociadas con la acumulación de almidón y azúcares en las hojas fuentes. Este proceso puede desencadenar un aumento en las concentraciones de ABA y especies reactivas de oxígeno-ROS en las hojas, proceso que genera cierre de estomas y por ende una afectación en la fotosíntesis por limitación estomática (Xu et al., 2014).

Caso contrario ocurre en la situación de la Figura 1b, donde la baja relación de órganos fuentes/vertedero, promueve que la carga de fotoasimilados se distribuya para garantizar la formación de los frutos, dado que son vertederos prioritarios, y de esta manera, se afecta el crecimiento de estructuras vegetativas y los patrones de distribución de los fotoasimilados. Esta afirmación anterior fue corroborada en el estudio de Li et al. (2015), donde conservando la carga de frutos estándar de tres cultivares de tomate (baja relación fuente/vertedero), la partición de biomasa fue modificada por la fuerza de vertedero ejercida por los frutos, registrando datos promedio de $38 \%$ en hojas, $16 \%$ tallo y $46 \%$ en frutos con respecto a plantas que tenían media carga (45\% hojas; $20 \%$ tallo y $35 \%$ frutos).

Por otro lado, durante esta situación de baja actividad de órganos fuentes, se ha reportado que hay incrementos en las tasas fotosintéticas. Glanz-Idan $\&$ Wolf (2020) reportan que las tasas fotosintéticas en plantas de tomate parcialmente defoliadas se incrementaron en un 35\%, y esto se debe a los aumentos en la conductancia estomática y a los contenidos de clorofilas que se registraron en estas plantas, para garantizar una mayor asimilación de $\mathrm{CO}_{2}$, y por tanto, aumentos en la producción de fotoasimilados. Además, se ha reportado que los frutos de tomate durante sus etapas tempranas de desarrollo, son órganos fotosintéticos y de esta manera pueden contribuir entre un 10 a 15\% de la producción de carbohidratos para su crecimiento y desarrollo (Osorio et al., 2014). Sin embargo, en una situación de baja relación fuente/vertedero es común evidenciar variación en los tamaños de los frutos, debido a la alta actividad de órganos vertederos (Ho, 1996; Aslani et al., 2020).

Quintana-Baquero et al. (2010), evaluaron el efecto de la baja relación fuente/ vertedero sobre el rendimiento y la calidad de los frutos cosechados de cultivares de larga vida. En este estudio se reguló la actividad de los frutos de tomate (racimos/planta) y el crecimiento apical y lateral del cultivar (podas). 


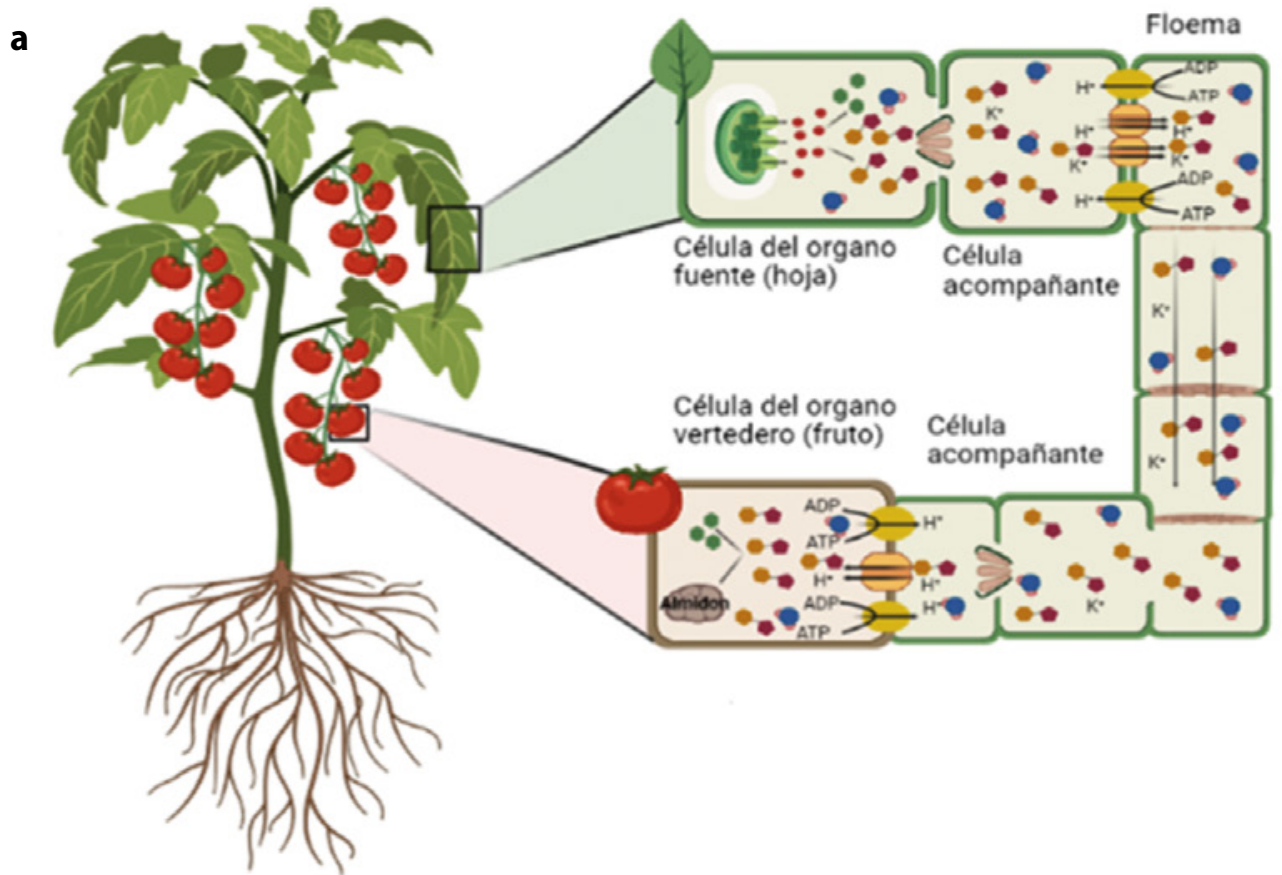

b

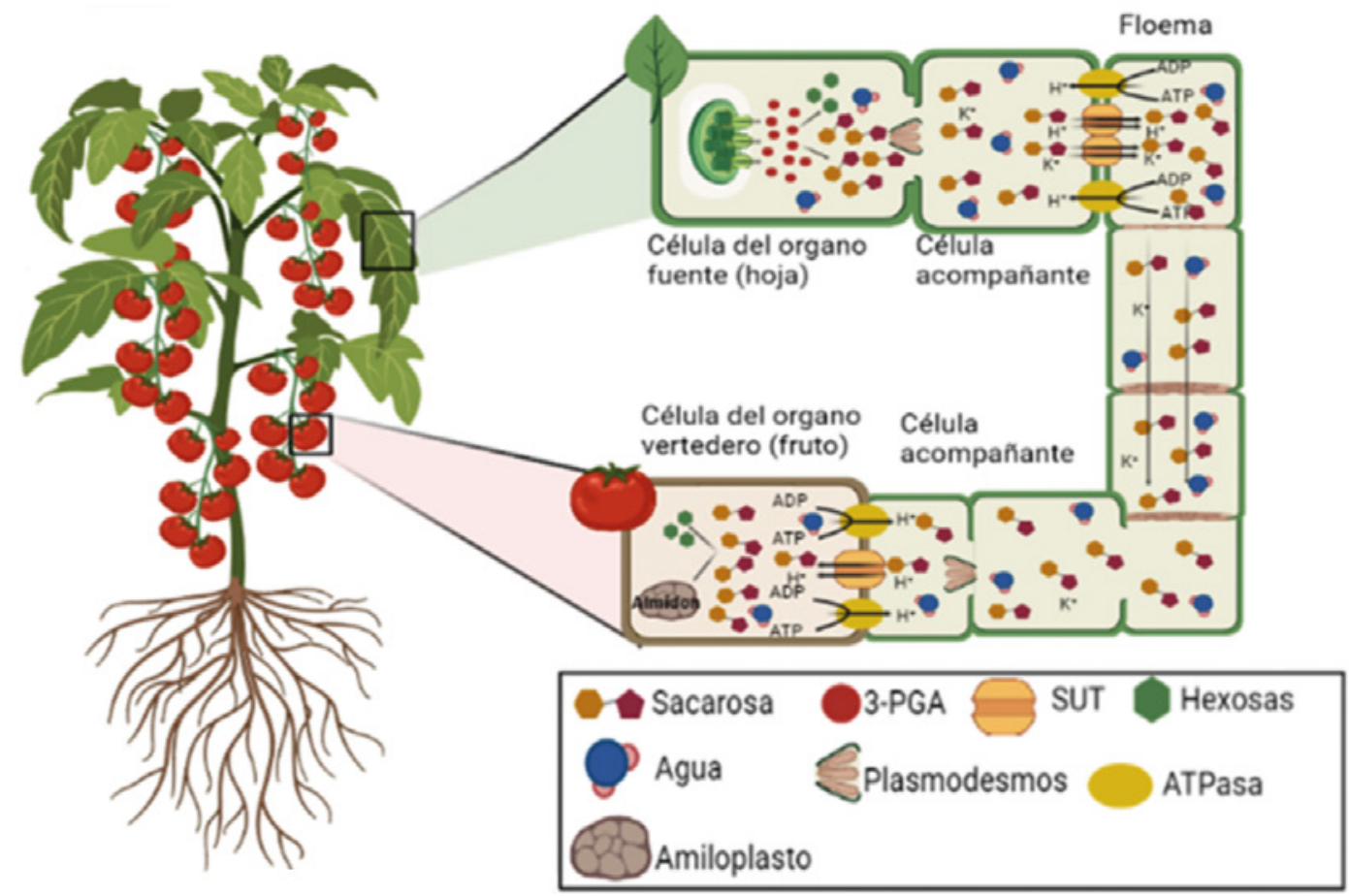

FIGURA 1. Efecto de la relación de órganos fuente/vertedero sobre la distribución de fotoasimilados en plantas de tomate (S. lycopersicum). 1a: condición de baja relación fuente/vertedero y $1 \mathrm{~b}$ : condición de alta relación fuente/vertedero.

Se obtuvo que conservando 12 racimos/planta, se alcanzó la mayor producción, no obstante, esto genero una disminución en la producción de frutos de mejor calidad (extra). Parte de la explicación de este resultado, se debe a que, con el aumento del número de frutos (fuerza de vertedero), se genera 
una competencia entre la distribución de los fotoasimilados entre los órganos vertederos y de esta manera, se reporta tamaños variables en los frutos dado que se presenta una baja relación fuente/vertedero, además de que se reduce la distribución de la biomasa hacia los órganos vegetativos, este mismo resultado también ha sido reportado por Kang et al. (2011).

\section{Condiciones de estrés hídrico}

Otro factor externo que influye en la relación y la fuerza de los órganos fuente/vertedero, y en la distribución de los fotoasimilados, es el déficit hídrico del suelo, el cual, promueve un estrés de tipo abiótico en las plantas (Rodrigues et al., 2019). El estrés hídrico en las plantas ha sido asociado como uno de los factores ambientales que afecta los rendimientos y el desempeño fisiológico de los cultivos de tomate (Chen et al., 2013; Medyouni et al., 2021; Luo et al., 2021). Una de las respuestas fisiológicas que afecta el estrés hídrico en las plantas es el cierre de las estomas, lo cual, limita la asimilación de $\mathrm{CO}_{2}$, disminuye la tasa de carboxilación, la producción de fotoasimilados y en general afecta la relación de órganos fuente/vertedero (Sun et al., 2020). En el cultivo de tomate se ha reportado que el estrés hídrico afecta la acumulación de fotoasimilados en los frutos debido a la baja actividad de las enzimas relacionadas en la síntesis de sacarosa y almidón (SuSy, almidón fosforilasa -SP, SPS y la adenosin difosfato glucosa pirofosforilasa- AGPasa (Luo et al., 2021). Esta reducción de la actividad de enzimas afecta directamente la fuerza de vertedero en los frutos, lo que disminuye la carga de fotoasimilados desde los órganos fuentes hacia los vertederos y así los rendimientos (Osorio et al., 2014).

En el estudio de Chen et al. (2013) se constató que el déficit hídrico puede afectar significativamente los rendimientos del cultivo de tomate. Sin embargo, se evidencia que el efecto del déficit hídrico en los rendimientos está relacionado con la etapa de crecimiento del cultivo, dado que ellos reportan efectos en los rendimientos cuando se disminuye el riego durante la etapa de floración, desarrollo y maduración del fruto. Aunque disminuye los rendimientos por el efecto del déficit hídrico, se reporta que se mejora la calidad, acumulando más contenidos de azúcares solubles (mayormente hexosas), vitamina $C$ y se mejora la firmeza de los frutos, y esto mismo ha sido reportado por Hou et al. (2020).

\section{Luz}

La radiación solar como fuente primaria para la producción de fotoasimilados por medio de los procesos fotosintéticos, tiene efectos directos sobre el balance de órganos fuentes/vertederos (Kinet, 1977; Hikosaka et al., 2013). Los Factores como intensidad y calidad de la radiación solar, han sido reportados 
sobre el efecto en la distribución y balance de fotoasimilados para el cultivo de tomate (Hikosaka et al., 2013; Ji et al., 2020; Wang et al., 2020). En el estudio de Wang et al. (2020), en el cual, se evaluaron diferentes porcentajes de sombreamiento sobre el desarrollo de brotes florales (órganos vertederos) en un cultivo de tomate, se determinó que al regular la intensidad de la luz, se afectan el número de brotes florales, la longitud de la inflorescencia y se prolonga la iniciación floral. Estos resultados evidencian que hubo una leve carga de fotoasimilados hacia los órganos vertederos (brotes florales), lo que retrasó la iniciación de la floración y generó el almacenamiento de almidón y azúcares solubles en las hojas fuentes.

En el estudio de Lanoue et al. (2018) evaluaron el efecto de la calidad del espectro de la longitud de onda (luz roja, azul, naranja, verde y blanca) sobre la tasa de transporte de fotoasimilados en el cultivo de tomate. En este estudio se determinó que no hubo efecto de las diferentes longitudes de onda sobre la tasa de exportación de fotoasimilados y que dicha exportación sigue el mismo patrón de las tasas fotosintéticas. Por otro lado, Ji et al. (2020) reportan que en plantas de tomate sometidas a radiación del rojo lejano aumentó la partición de masa seca, la tasa de crecimiento en los frutos y aumento en un 38\% la fuerza de vertedero. Este efecto fue asociado a la expresión de los genes involucrados en el transporte de sacarosa y del metabolismo de azúcares lo que incidió en un aumento en la fuerza de vertedero del fruto. También en este mismo estudio, se registra incremento significativo en el contenido de fructosa y glucosa en los frutos, además de que la maduración fue acelerada en las plantas sometidas a este espectro de la luz.

\section{Estado nutricional}

Así como los factores mencionados anteriormente, las deficiencias nutricionales pueden afectar el metabolismo del carbono y la translocación y distribución de los fotoasimilados en los órganos vertederos (Clanz-Idan \& Wolf., 2020). En plantas de tomate no fertilizadas, se ha reportado que disminuye las tasas fotosintéticas, las medidas de intercambio gaseoso (conductancia estomática y transpiración) y la concentración de las clorofilas, lo que disminuye la fuerza de los órganos fuentes por la baja producción de fotoasimilados (Kanai et al., 2007; Glanz-Idan \& Wolf., 2020). Luo et al. (2021) reportaron que las deficiencias de potasio en plantas de tomate redujeron la fuerza de vertedero de los frutos, debido a la baja actividad de enzimas vinculadas en el metabolismo de azúcares (Susy, SPS, Al y AGPasa), lo que reduce la carga de fotoasimilados distribuida hacia los frutos. Además, se reporta que la deficiencia de potasio disminuye entre un $53 \%$ a $72 \%$ la partición de la masa seca en los órganos de las plantas de tomate y esto se debe a que el $\mathrm{K}^{+}$tiene un efecto directo sobre el crecimiento y desarrollo de la planta, así como en 
la tasa de transporte de los fotoasimilados en los frutos, reduciendo así el tamaño de estos órganos y la fuerza de vertedero (Kanai et al., 2007).

\section{Papa (S. tuberosum L.)}

La papa constituye el tercer alimento de mayor consumo después del maíz y el trigo, considerándose un cultivo de gran importancia en la seguridad alimentaria e industrial a nivel mundial (De Jong, 2016; Gómez, 2018). En papa, las hojas y tallos constituyen los tejidos fuentes de la planta, generadores de fotoasimilados, con capacidad de exportar, siendo una función integral basada en procesos de fotosíntesis, respiración y crecimiento (Castellanos et al., 2010; Golovko \& Tavalenkova, 2019). Este cultivo tiene una gran actividad de órganos vertederos durante la fase reproductiva como los estolones, tubérculos, flores y frutos, y esta condición estimula diferentes mecanismos para que la planta sea eficiente en el transporte y utilización de los fotoasimilados (Abelenda et al., 2020). Sin embargo, los tubérculos constituyen los vertederos prioritarios y el órgano de interés económico para este cultivo (Castellanos et al., 2010; De Jong, 2016).

\section{Transporte de fotoasimilados en órganos vertederos}

El transporte de fotoasimilados en la planta de papa tiene los mismos mecanismos (simplastica y apoplastica) de translocación de fotoasimilados reportados en el capítulo de tomate (Aliche et al., 2020), no obstante, se puede presentar modificaciones en los mecanismos de transporte, según la etapa fenológica del cultivo (Golovko \& Tavalenkova, 2019; Abelenda et al., 2020). En la Figura 2, se simuló como los patrones de transporte y distribución de los fotoasimilados obtenidos en los órganos fuentes (hojas), se modifican de acuerdo con la etapa fenológica del cultivo, por ejemplo, durante el inicio de la tuberización y floración (órganos vertederos), el transporte de fotoasimilados se direccionan para ambos órganos, aunque se distribuye más hacia los estolones donde se lleva a cabo la diferenciación celular y el inicio del crecimiento y desarrollo de los tubérculos por ser vertederos prioritarios, además de generar mayor fuerza para utilizar y metabolizar los fotoasimilados (Ñústez et al., 2009; Castellanos et al., 2010). Recientemente, se ha reportado que los incrementos en la concentración de sacarosa actúan como molécula señalizadora en los procesos de inducción de la floración e inicio de la tuberización, y de esta manera, se da inicio a la regulación de la distribución de fotoasimilados hacia estos órganos (Abelenda et al., 2019). También se ha postulado que las proteínas SP6A tiene roles claves en la formación de los tubérculos (Lehretz et al., 2019).

Posteriormente durante el llenado o desarrollo de los tubérculos, cambia la distribución de fotoasimilados (tubérculos y frutos), de hecho, se reporta 
que, durante esta etapa fenológica, la concentración puede distribuirse hasta en un $90 \%$ en los tubérculos (Golovko \& Tavalenkova, 2019). También en la Figura 2, se ilustra de acuerdo con el estudio de Abelenda et al. (2019) que, durante el llenado de los tubérculos, se ha determinado la síntesis de proteínas StSP6A en las hojas, las cuales, se translocan hacia los tubérculos, donde al unirse a los transportadores de membrana StSWEET11, bloquean la descarga para el transporte de sacarosa vía apoplastica, cambiando el modo de descarga a vía simplastica (Abelenda et al., 2019).

Por otro lado, Golovko \& Tavalenkova (2019) determinaron los patrones de transporte de fotoasimilados según la posición de las hojas (5, 10 y 15) para el desarrollo de la planta y de los tubérculos. Estos autores encontraron que en plantas jóvenes el tercio inferior de las hojas (hoja 5) distribuye en un 95\% los fotoasimilados hacia tallos y raíces y solo $5 \%$ es transportado hacia el meristemo apical. Durante el inicio de la tuberización, todas las hojas distribuyeron en su mayoría los fotoasimilados hacia los tubérculos, sin embargo, de acuerdo con la posición de las hojas, esta distribución es diferencial. Para

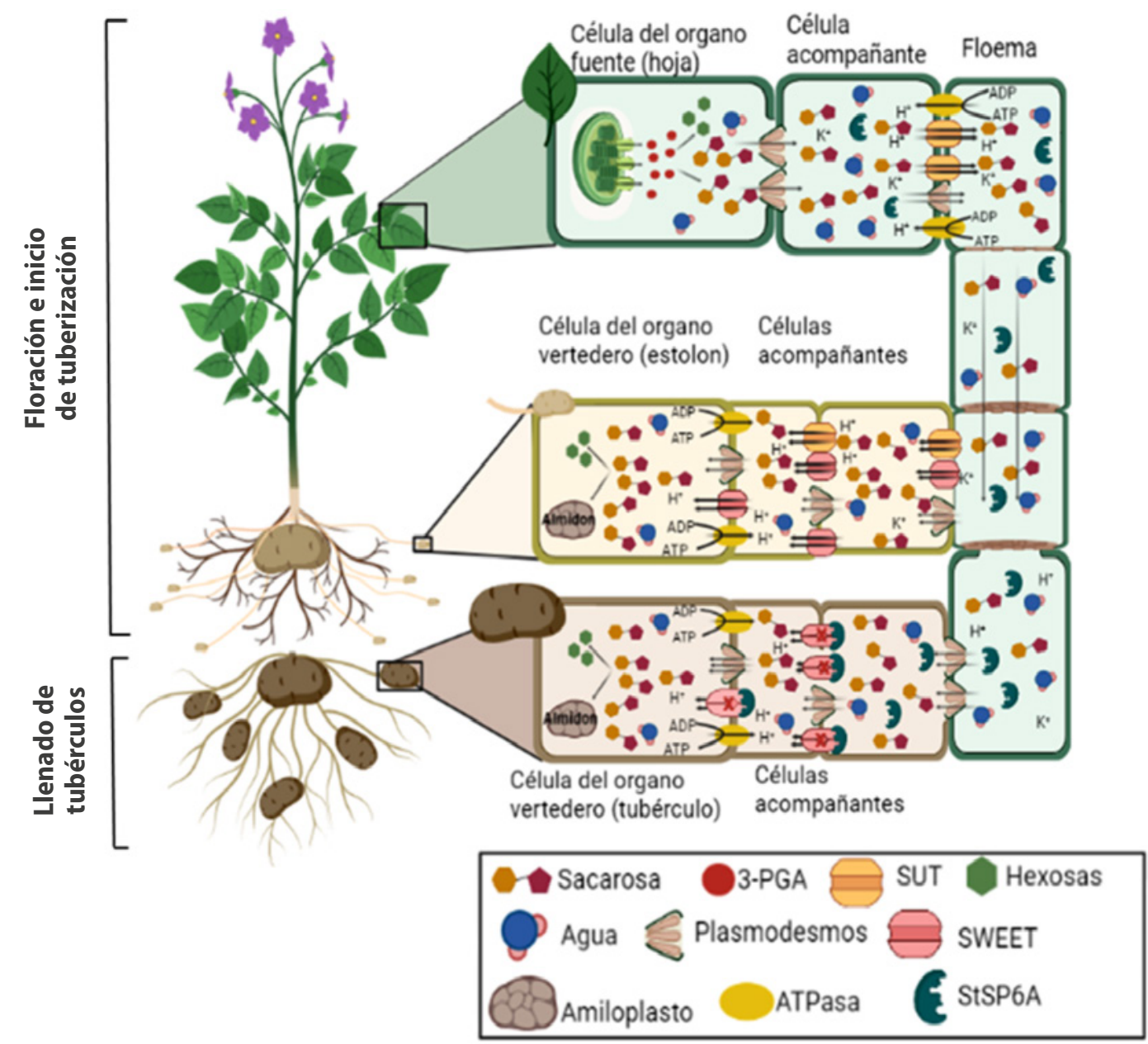

FIGURA 2. Distribución de fotoasimilados durante el inicio del desarrollo y llenado de los tubérculos en plantas de papa. 
las hojas ubicadas en el tercio inferior el $90 \%$ de los fotoasimilados fueron translocados hacia los estolones y tubérculos, mientras que las hojas ubicadas en el tercio medio (hoja 10) se distribuyó el 60\% hacia los tubérculos y el restante fue asignado al mantenimiento de procesos fisiológicos (respiración, síntesis de pared celular y síntesis de esqueletos carbonados). Por último, se distribuyó el $90 \%$ de los fotoasimilados producidos en las hojas ubicadas en el tercio superior (hoja 15) hacia el desarrollo de los tubérculos.

\section{Factores que influyen en la distribución de fotoasimilados en los órganos vertederos}

\section{Relación fuente/vertedero}

La partición de carbono producido en los procesos fotosintéticos de las plantas depende en gran medida de la relación fuente/vertedero y la fuerza de los vertederos para translocar los azúcares (Castellanos et al., 2010; Aliche et al., 2020). Aunque la distribución de los fotoasimilados parece ser controlada, esta depende del cultivar (Ñústez et al., 2009; Castellanos et al., 2010). En papa se ha relacionado la fuerza del vertedero en relación al tamaño de los tubérculos, referido como el peso seco y la tasa relativa del crecimiento. Mediante esta forma de analizar la influencia en la relación fuente/vertedero, se determinó que durante la etapa de llenado de los tubérculos diferentes cultivares (Diacol Capiro, Pastusa suprema, Betina y Esmeralda) presentaron limitación en órganos fuentes, lo cual, genero rendimientos diferenciales entre 45,000 a 55,000 kg ha-1 (Castellanos et al., 2010).

En el estudio de Caicedo et al. (2010) se evaluó diferentes niveles de defoliación (baja relación fuente/vertedero) aplicados en diferentes etapas fenológicas del cultivo de papa. Se determinó que las plantas defoliadas en un $40 \%$ durante la formación de los tubérculos se afectaron los rendimientos. Este efecto se debe por el desbalance en los órganos fuente y por tanto disminución en la producción de los fotoasimilados, lo que conlleva a generar una baja actividad de vertederos. Además, durante esta etapa fenológica, se requiere una gran cantidad de transporte de fotoasimilados hacia los tubérculos, dado que hay una mayor fuerza de vertedero para satisfacer las necesidades en los incrementos en las tasas de división y elongación celular de estos órganos. Lo anterior, se constata con los aumentos progresivos y partición de la biomasa reportados en los estudios de Ñústez et al. (2009) y Castellanos et al. (2010).

Por otro lado, en el cultivo de papa también se ha evidenciado que el índice de cosecha; parámetro que relaciona el peso seco total de los órganos de interés económico con el peso total de la planta (Victorio et al., 1986; Ñústez et al., 2009; Kim and Lee, 2019), y puede reflejar los patrones de distribución de los fotoasimilados. Este parámetro indica la actividad de los órganos vertederos 
en una determinada etapa fenológica del cultivo, y de esta manera, entre mayor sea su valor se puede interpretar la proporción de los fotoasimilados direccionada hacia los tubérculos. Sin embargo, este parámetro está relacionado con el tipo de cultivar (Victorio et al., 1986), por ejemplo, en cultivares como Diacol Capiro, Betina y esmeralda se reporta un índice de cosecha entre $80 \%$ a $85 \%$ durante la etapa de llenado de los tubérculos, en comparación con el cultivar Pastusa Suprema que alcanza un valor del $72 \%$ (Ñústez et al., 2009). Estos valores permiten concluir que la actividad de vertederos en los cultivares Diacol Capiro, Betina y esmeralda tienen una mayor capacidad de importar, utilizar y metabolizar los fotoasimilados generados en los órganos fuentes.

\section{Condiciones de estrés hídrico}

En papa como en la mayoría de las plantas bajo condiciones de estrés hídrico se prioriza la partición de fotoasimilados hacia las raíces, como mecanismo de evitación, para aumentar la capacidad de exploración de este recurso en el suelo (Aliche et al., 2020; Brunner et al., 2015). Bajo estas condiciones, se ha reportado que se afecta la relación de los órganos fuentes/vertederos de interés comercial, reduciéndose hasta en $17 \%$ el número de tubérculos, disminuciones significativas en el número de hojas y altura de las plantas (Deblonde \& Ledent, 2001). Caso contrario ocurrió en lo encontrado por Rodríguez et al. (2016), donde se reporta que no hubo reducciones significativas en las variables de número de hojas/tallo, número de tallos/sitio y longitud del tallo; componentes de rendimiento de este cultivo. Mientras que, si se registró una reducción en las etapas fenológicas como la floración, desarrollo foliar y maduración de los frutos. Esta respuesta fue asociada a la plasticidad de la planta de papá como un mecanismo para adaptarse a las condiciones de estrés hídrico. Sin embargo, cuando el estrés es más severo, hay reducciones de hasta el 79\% en los rendimientos (Deblonde \& Ledent, 2001; Aliche et al., 2020).

De otra parte, Aliche et al. (2020) reportan que en plantas con estrés hídrico hay disminuciones en los contenidos de almidón en los tubérculos e incrementos en los contenidos de sacarosa en hojas fuente. Esto se debe a la disminución de la actividad de enzimas vinculadas al metabolismo de azucares (baja fuerza de vertedero) y a la baja actividad de transportadores de sacarosa. Lerna \& Mauromicale (2012), observaron una disminución significativa en el número y tamaño de las hojas, bajas tasas fotosintéticas y como consecuencia limita la formación, el tamaño y rendimiento de los tubérculos, este mismo resultado lo obtuvieron Li et al. (2016).

\section{LuZ}

La fotosíntesis es limitada por la intensidad y calidad de la luz, lo que también afecta la capacidad de translocación de fotoasimilados hacia los órganos 
vertederos, y por tanto, los patrones de distribución de masa seca de los diferentes órganos de la planta de papa (Zheng et al., 2009; Li et al., 2020). En este sentido, regulando la intensidad de la luz en plantas de papa se determinaron diferencias significativas en la distribución de la masa seca total en los diferentes órganos (hojas, tallo y tubérculos), reportando una reducción del $25 \%$ en comparación con las plantas control. Así mismo, se reportan diferencias significativas en la acumulación de azúcares como hexosas y almidón en los tubérculos.

En cuanto al efecto de la calidad de la luz, en el estudio de Li et al. (2020) se encontró que hay una modificación en los patrones de distribución de la masa seca en los diferentes órganos de la planta. También se reporta que la luz roja y azul presentaron mayor acumulación de masa seca en los microtubérculos (entre $75 \%$ a $80 \%$ ) con respecto a la luz amarilla y verde (55\% a $65 \%$ ), lo que indica que los espectros de longitud de onda de estos tipos de luz afectan la distribución de fotoasimilados en los órganos vertederos y favorece la acumulación en los órganos fuentes. Este mismo resultado lo obtuvo Chen et al. (2019), donde la calidad de luz azul presenta mayor área foliar y contenidos de pigmento respecto a las plantas tratadas con luz blanca (control), lo que favoreció mayor fuerza de los órganos fuente y así se obtuvo una temprana inducción a la tuberización y mayor número de tubérculos.

\section{Estado nutricional}

La disponibilidad de nutrientes regula la relación de los órganos fuente/vertedero y por tanto afecta la distribución de los fotoasimilados y productividad del cultivo de papa (Li et a., 2016; Gómez et al., 2017; Grzebisz et al., 2020). Lo anterior fue corroborado en plantas con deficiencias en nitrógeno, dado que estas condiciones redujeron significativamente el área foliar, y por tanto las tasas fotosintéticas de las plantas, lo que conllevo a disminuir el suministro de los fotoasimilados por los órganos fuente hacia los órganos vertederos y de esta manera, se tuvo limitaciones en los rendimientos de los cultivos (Li et al., 2016). También, se ha reportado que disminuciones o excesos de nitrógeno en la fertilización del cultivo de papa, puede afectar la partición de fotoasimilados, principalmente por reducción del crecimiento del dosel ocasionando senescencia prematura de las hojas y disminución de la translocación de los fotoasimilados en etapas de llenado. Así mismo, se mencionan reducciones en las tasas fotosintéticas, en la superficie total de la hoja y en el número y peso de los tubérculos (Ruža et al., 2013; Gómez et al., 2017). 


\section{Conclusiones y perspectivas}

La distribución de fotoasimilados en cultivos de tomate y papa es un proceso integral, el cual considera optimizar la producción de fotoasimilados por los órganos fuentes, para mejorar la eficiencia en la utilización de estos productos en los órganos vertederos de interés comercial. De esta manera, garantizar la relación adecuada de fuente/vertedero se convierte en una herramienta necesaria para favorecer el rendimiento y la calidad de los frutos de tomate, y tubérculos de papa. Sin embargo, en esta revisión se discute que mantener este balance de fuente/vertedero resulta ser afectada por diversos factores como estrés hídrico, intensidad y calidad de la luz, deficiencias nutricionales y factores internos como la fuerza de los órganos fuente/vertedero. Aunque existen evidencias positivas del efecto del déficit de riego en algunas etapas fenológicas y también en el uso de tecnologías para mejorar la calidad de la luz sobre los rendimientos y calidad de los órganos de interés comercial tanto para tomate y papa, es necesario realizar más investigaciones sobre estos temas enfocadas en las condiciones particulares del trópico.

\section{Referencias}

Abelenda, J. A., Bergonzi, S., Oortwijn, M., Sonnewald, S., Du, M., Visser, R. G. F., Sonnewald, U., \& Bachem, C. W. B. (2019). Source-Sink Regulation Is Mediated by Interaction of an FT Homolog with a SWEET Protein in Potato. Current Biology, 29(7), $1178-1186 . e 6$. https://doi.org/10.1016/j.cub.2019.02.018.

Agronet. (2019). Área, producción y rendimiento nacional por cultivo. https://www. agronet.gov.co/estadistica/Paginas/home.aspx?cod=1 (consultado septiembre, 2021).

Aliche E., Theeuwen T., Oortwijn M., Visser R., Linden C., 2020. Carbon partitioning mechanisms in Potato under drought stress. Plant Physiology and Biochemistry, 146, 211-219. https://doi.org/10.1016/j.plaphy.2019.11.019.

Aluko, O. O., Li, C., Wang, Q., \& Liu, H. (2021). Sucrose Utilization for Improved Crop Yields: A Review Article. International Journal of Molecular Sciences, 22(9), 4704. https://doi.org/10.3390/ijms22094704.

Ardila, G. A., Fischer, G., \& Balaguera-López, H. E. (2011). Caracterización del crecimiento del fruto y producción de tres híbridos de tomate (Solanum lycopersicum L.) en tiempo fisiológico bajo invernadero. Revista Colombiana de Ciencias Hortícolas, 5(1), 44-56. https://doi.org/10.17584/rcch.2011v5i1.1252

Aslani, L., Gholami, M., Mobli, M., \& Sabzalian, M. R. (2020). The influence of altered sink-source balance on the plant growth and yield of greenhouse tomato. Physiology and Molecular Biology of Plants, 26(1 1), $2109-2123$. https://doi. org/10.1007/s12298-020-00891-2.

Balaguera-López, H. E., Álvarez-Herrera, J. G., Martínez-Arévalo, G. E., \& Balaguera, W. A. (2009). El contenido de arcilla del suelo influye en el rendimiento de un cultivo de tomate (Solanum lycopersicum L.). Revista Colombiana de Ciencias Hortícolas, 3(2), 199-209. https://doi.org/10.17584/rcch.2009v3i2.1213 
Bihmidine, S., Hunter, C. T., Johns, C. E., Koch, K. E., \& Braun, D. M. (2013). Regulation of assimilate import into sink organs: Update on molecular drivers of sink strength. Frontiers in Plant Science, 4. https://doi.org/10.3389/fpls.2013.00177.

Brunner, I., Herzog, C., Dawes, M. A., Arend, M., \& Sperisen, C. (2015). How tree roots respond to drought. Frontiers in Plant Science, 6, 547. https://doi. org/10.3389/fpls.2015.00547.

Caicedo, D. R., Tellez, M. S. R., Molano, L. E. R., \& López, C. E. Ñ. (2010). Efecto de Diferentes Niveles y Épocas de Defoliación Sobre el Rendimiento de la Papa (Solanum tuberosum cv. Parda Pastusa). Revista Facultad Nacional de Agronomía - Medellín, 63(2), 5521-5531.

Castellanos, M. S., Abril, M. S., \& López, C. E. Ñ. (2010). Análisis de Crecimiento y Relación Fuente-Demanda de Cuatro Variedades de Papa (Solanum tuberosum L.) en el Municipio de Zipaquirá (Cundinamarca, Colombia). Revista Facultad Nacional de Agronomía - Medellín, 63(1), 5253-5266.

Carpaneto, A., Geiger, D., Bamberg, E., Sauer, N., Fromm, J., \& Hedrich, R. (2005). Phloem-localized, Proton-coupled Sucrose Carrier ZmSUT1 Mediates Sucrose Efflux under the Control of the Sucrose Gradient and the Proton Motive Force *. Journal of Biological Chemistry, 280(22), 21437-21443. https://doi.org/10.1074/jbc. M501785200

Córdoba-Novoa, H. A., Gómez, S. V., \& Ñústez, C. E. (2018). Evaluación del rendimiento y fenología de tres genotipos de tomate cherry (Solanum lycopersicum L.) bajo condiciones de invernadero. Revista Colombiana de Ciencias Hortícolas, 12(1), 113-125. https://doi.org/10.17584/rcch.2018v12i1.7348

Chang, T.-G., Zhu, X.-G., \& Raines, C. (2017). Source-sink interaction: A century old concept under the light of modern molecular systems biology. Journal of Experimental Botany, 68(16), 4417-4431. https://doi.org/10.1093/jxb/erx002.

Chen, J., Kang, S., Du, T., Qiu, R., Guo, P., \& Chen, R. (2013). Quantitative response of greenhouse tomato yield and quality to water deficit at different growth stages. Agricultural Water Management, 129, 152-162. https://doi.org/10.1016/j. agwat.2013.07.011.

Chen, L., Yang, Y., Jiang, Y., Zhao, J., Zang, H., Wang, X., Hu, Y., \& Xue, X. (2019). RNA-Seq Analysis Reveals Differential Responses of Potato (Solanum tuberosum L.) Plantlets Cultured in vitro to Red, Blue, Green, and White Light-emitting Diodes (LEDs). Journal of Plant Growth Regulation, 38(4), 1412-1427. https://doi. org/10.1007/s00344-019-09944-7

De Jong, H., 2016. Impact of the potato on society. Am. J. Potato Res., 93(5), 415 429. https://doi.org/10.1007/s12230-016-9529-1.

Deblonde, P. M. K., \& Ledent, J. F. (2001). Effects of moderate drought conditions on green leaf number, stem height, leaf length and tuber yield of potato cultivars. European Journal of Agronomy, 14(1), 31-41. https://doi.org/10.1016/ S1161-0301(00)00081-2.

Federación Colombiana de Productores de Papa-Fedepapa. (2020). Nuestros héroes de campo no paran frente al Covid-19. Revista papa, 50, 1-14. https://fedepapa.com/wp-content/uploads/2020/04/REVISTA-50-completa-2.pdf.

Fischer, G., Almanza-Merchán, P. J., \& Ramírez, F. (2012). Source-sink relationships in fruit species: A review. Revista Colombiana de Ciencias Hortícolas, 6(2), 238253. https://doi.org/10.17584/rcch.2012v6i2.1980 
Glanz-Idan, N., \& Wolf, S. (2020). Upregulation of photosynthesis in mineral nutrition-deficient tomato plants by reduced source-to-sink ratio. Plant Signaling \& Behavior, 15(2), 1712543. https://doi.org/10.1080/15592324.2020.1712543.

Golovko, T.K., Tabalenkova, G.N., 2019. Source-Sink Relationships in Potato Plants. Russ J Plant Physiol 66, 664-671. https://doi.org/10.1134/ S1021443719030051.

Gómez M., Magnitskiy, S., Rodríguez, A. Darghan. (2017). Accumulation of N, P, and $\mathrm{K}$ in the tubers of potato (Solanum tuberosum L. ssp. Andigena) under contrasting soils of the Andean region of Colombia. Agronomía Colombiana. 35. 59-67. https://doi.org/10.15446/agron.colomb.v35n1.61068.

Grzebisz, W., Szczepaniak, W., \& Bocianowski, J. (2020). Potassium fertilization as a driver of sustainable management of nitrogen in potato (Solanum tuberosum L.). Field Crops Research, 254, 107824. https://doi.org/10.1016/j.fcr.2020.107824

Ho, L. C. (1996). The mechanism of assimilate partitioning and carbohydrate compartmentation in fruit in relation to the quality and yield of tomato. Journal of Experimental Botany, 47, 1239-1243.

Ho, L.-H., Klemens, P. A. W., Neuhaus, H. E., Ko, H.-Y., Hsieh, S.-Y., \& Guo, W.-J. (2019). SISWEET1a is involved in glucose import to young leaves in tomato plants. Journal of Experimental Botany, 70(12), 3241-3254. https://doi.org/10.1093/jxb/ erz154

Hou, X., Zhang, W., Du, T., Kang, S., \& Davies, W. J. (2020). Responses of water accumulation and solute metabolism in tomato fruit to water scarcity and implications for main fruit quality variables. Journal of Experimental Botany, 71(4), 1249-1264. https://doi.org/10.1093/jxb/erz526

Hikosaka, S., lyoki, S., Hayakumo, M., \& Goto, E. (2013). Effects of light intensity and amount of supplemental LED lighting on photosynthesis and fruit growth of tomato plants under artificial conditions. Journal of Agricultural Meteorology, 69 (2), 93-100 https://doi.org/10.2480/AGRMET.69.2.5

Ji, Y., Ocaña, D. N., Choe, D., Larsen, D. H., Marcelis, L. F. M., \& Heuvelink, E. (2020). Far-red radiation stimulates dry mass partitioning to fruits by increasing fruit sink strength in tomato. New Phytologist, 228(6), 1914-1925. https://doi. org/10.1111/nph.16805.

Kanai, S., Ohkura, K., Adu-Gyamfi, J. J., Mohapatra, P. K., Nguyen, N. T., Saneoka, H., \& Fujita, K. (2007). Depression of sink activity precedes the inhibition of biomass production in tomato plants subjected to potassium deficiency stress. Journal of Experimental Botany, 58(11), 2917-2928. https://doi.org/10.1093/ jxb/erm 149

Kang, M., Yang, L., Zhang, B., \& de Reffye, P. (2011). Correlation between dynamic tomato fruit-set and source-sink ratio: A common relationship for different plant densities and seasons? Annals of Botany, 107(5), 805-815. https://doi. org/10.1093/aob/mcq244.

Kinet, J. M. (1977). Effect of light conditions on the development of the inflorescence in tomato. Scientia Horticulturae, 6(1), 15-26. https://doi. org/10.1016/0304-4238(77)90074-7

Lanoue, J., Leonardos, E. D., \& Grodzinski, B. (2018). Effects of Light Quality and Intensity on Diurnal Patterns and Rates of Photo-Assimilate Translocation and 
Transpiration in Tomato Leaves. Frontiers in Plant Science, 9. https://doi. org/10.3389/fpls.2018.00756.

Lehretz, G. G., Sonnewald, S., Hornyik, C., Corral, J. M., \& Sonnewald, U. (2019). Post-transcriptional Regulation of FLOWERING LOCUS T Modulates Heat-Dependent Source-Sink Development in Potato. Current Biology, 29(10), 1614-1624. e3. https://doi.org/10.1016/j.cub.2019.04.027.

Lerna, A., \& Mauromicale, G. (2012). Tuber yield and irrigation water productivity in early potatoes as affected by irrigation regime. Agricultural Water Management, 115, 276-284. https://doi.org/10.1016/j.agwat.2012.09.011

Li, T., Heuvelink, E., \& Marcelis, L. F. M. (2015). Quantifying the source-sink balance and carbohydrate content in three tomato cultivars. Frontiers in Plant Science, 6, 416. https://doi.org/10.3389/fpls.2015.00416

Li, W., Xiong, B., Wang, S., Deng, X., Yin, L., \& Li, H. (2016). Regulation Effects of Water and Nitrogen on the Source-Sink Relationship in Potato during the Tuber Bulking Stage. Plos One, 11(1), e0146877. https://doi.org/10.1371/journal. pone.0146877

Li, R., You, J., Miao, C., Kong, L., Long, J., Yan, Y., Xu, Z., \& Liu, X. (2020). Monochromatic lights regulate the formation, growth, and dormancy of in vitrogrown Solanum tuberosum L. microtubers. Scientia Horticulturae, 261, 108947. https://doi.org/10.1016/j.scienta.2019.108947

López-Delacalle, M., Camejo, D., Garcia-Marti, M., Lopez-Ramal, M. J., Nortes, P. A., Martinez, V., \& Rivero, R. M. (2021). Deciphering fruit sugar transport and metabolism from tolerant and sensitive tomato plants subjected to simulated field conditions. Physiologia Plantarum, 1-14. https://doi.org/10.1111/ppl.13355

Luo, A., Zhou, C., \& Chen, J. (2021). The Associated With Carbon Conversion Rate and Source-Sink Enzyme Activity in Tomato Fruit Subjected to Water Stress and Potassium Application. Frontiers in Plant Science, 12, 681145 . https://doi. org/10.3389/fpls.2021.681145.

Medyouni, I., Zouaoui, R., Rubio, E., Serino, S., Ahmed, H. B., \& Bertin, N. (2021). Effects of water deficit on leaves and fruit quality during the development period in tomato plant. Food Science \& Nutrition, 9(4), 1949-1960. https://doi. org/10.1002/fsn3.2160

Mengel, K., \& Viro, M. (1974). Effect of Potassium Supply on the Transport of Photosynthates to the Fruits of Tomatoes (Lycopersicon esculentum). Physiologia Plantarum, 30(4), 295-300. https://doi.org/10.1111/j.1399-3054.1974. tb03660.x.

Maboko, M. M., Plooy, C. P. D., \& Chiloane, S. (2017). Yield of determinate tomato cultivars grown in a closed hydroponic system as affected by plant spacing. Horticultura Brasileira, 35, 258-264. https://doi.org/10.1590/S0102-053620170217

Mbonihankuye, C., Kusolwa, P., \& Msogoya, T. J. (2013). Assessment of the effect of pruning systems on plant developmental cycle - yield and quality of selected indeterminate tomato lines. Acta Horticulturae, 1007, 535-542. https://doi. org/10.17660/ActaHortic.2013.1007.61

Ñústez L, C. E. Ñ., Castellanos, M. S., \& Abril, M. S. (2009). Acumulación y distribución de materia seca de cuatro variedades de papa (Solanum tuberosum L.) en Zipaquirá, Cundinamarca (Colombia). Revista Facultad Nacional de Agronomía Medellín, 62(1), 4823-4834. 
Osorio, S., Ruan, Y.-L., \& Fernie, A. R. (2014). An update on source-to-sink carbon partitioning in tomato. Frontiers in Plant Science, 5, 516. https://doi.org/10.3389/ fpls.2014.00516

Perilla, A., Rodríguez, L. F., \& Bermúdez, L. T. (2011). Estudio técnico-económico del sistema de producción de tomate bajo invernadero en Guateque, Sutatenza y Tenza (Boyacá). Revista Colombiana de Ciencias Hortícolas, 5(2), 279-294. https://doi.org/10.17584/rcch.2011v5i2.1269

Smith, M. R., Rao, I. M., \& Merchant, A. (2018). Source-Sink Relationships in Crop Plants and Their Influence on Yield Development and Nutritional Quality. Frontiers in Plant Science, 9, 1889. https://doi.org/10.3389/fpls.2018.01889

Rodrigues, J., Inzé, D., Nelissen, H., \& Saibo, N. J. M. (2019). Source-Sink Regulation in Crops under Water Deficit. Trends in Plant Science, 24(7), 652-663. https:// doi.org/10.1016/j.tplants.2019.04.005

Rodríguez P, L., Sanjuanelo C., D., Ñústez L., C. E., \& Moreno-Fonseca, L. P. (2016). Growth and phenology of three Andean potato varieties (Solanum tuberosum L.) under water stress. Agronomía Colombiana, 34(2), 141-154. https://doi. org/10.15446/agron.colomb.v34n2.55279

Ruža, A., Skrabule, I., \& Vaivode, A. (2013). Influence of Nitrogen on Potato Productivity and Nutrient Use Efficiency. Proceedings of the Latvian Academy of Sciences. Section B. Natural, Exact, and Applied Sciences., 67(3), 247-253. https:// doi.org/10.2478/prolas-2013-0043

Wang, R., Gui, Y., Zhao, T., Ishii, M., Eguchi, M., Xu, H., Li, T., \& Iwasaki, Y. (2020). Determining the Relationship between Floral Initiation and Source-Sink Dynamics of Tomato Seedlings Affected by Changes in Shading and Nutrients. HortScience, 55(4), 457-464. https://doi.org/10.21273/HORTSCI14753-19.

Quintana-Baquero, R. A. Q., Balaguera-López, H. E., Herrera, J. G. Á., Hernández, J. F., \& Pinzón, H. H. (2010). Efecto del número de racimos por planta sobre el rendimiento de tomate (Solanum lycopersicum L.). Revista Colombiana de Ciencias Hortícolas, 4(2), 185-198. https://doi.org/10.17584/rcch.2010v4i2.1240.

Xu, M., Duan, W., Fan, P. G., Wu, B. H., Wang, L. J., Ma, L., Archbold, D. D., \& Li, S. H. (2014). Low sink-induced stomatal closure alters photosynthetic rates of source leaves in beans as dependent on $\mathrm{H}_{2} \mathrm{O}_{2}$ and $A B A$ accumulation in guard cells. Russian Journal of Plant Physiology, 61 (3), 397-408. https://doi.org/10.1134/ S1021443714020186.

Yoshioka, H. (1986). Translocation and distribution of photosynthates in tomato plants. Japan Agricultural Research Quarterly 19(4), 266-270.

Zheng, X., Jitsuyama, Y., Terauchi, T., \& Iwama, K. (2009). Effects of Drought and Shading on Non-structural Carbohydrate Stored in the Stem of Potato (Solanum tuberosum L.). Plant Production Science, 12(4), 449-452. https://doi. org/10.1626/pps.12.449 
